\title{
AVALIAÇÃO DA CAPACIDADE LIPOLÍTICA E PROTEOLÍTICA DE PSEUDOMONAS ISOLADAS DURANTE A ORDENHA MECÂNICA E MANUAL E NA ESTOCAGEM DO LEITE CRU REFRIGERADO
}

\author{
EVALUATION OF LIPOLYTIC AND PROTEOLYTIC ACTIVITY OF PSEUDOMONAS \\ ISOLATED DURING MILKING MECHANICAL AND MANUAL AND STORAGE OF \\ REFRIGERATED RAW MILK
}

F. BORTOLETTO ${ }^{1}$, A. M. C. VIDAL ${ }^{2}$, A. SARAN NETTO ${ }^{3}$, A. C. N. VAZ ${ }^{4}$, E. CAPODIFÓGLIO ${ }^{5}$, C. E. G. AGUILAR ${ }^{6}$, G. A. M. ROSSI ${ }^{6}$

\begin{abstract}
RESUMO
O objetivo do trabalho foi avaliar a atividade lipolítica e proteolítica em placas de Pseudomonas spp. isoladas durante a obtenção e armazenamento do leite cru refrigerado de propriedades com sistema de ordenha manual e mecânica, em períodos de chuva e seca. Os isolados de Pseudomonas spp. foram obtidos a partir de suabe de superfícies e equipamentos de ordenha e a partir de amostras de leite durante o armazenamento em tanque de expansão. Para a atividade lipolítica e proteolítica, os isolados foram semeados em Ágar Tributirina e Ágar Leite, respectivamente. Foram comparadas as porcentagens de presença de isolados em tipo de ordenha e período do ano. A atividade lipolítica foi verificada apenas no período da seca, sobressaindo a ordenha manual com melhor condição para o não desenvolvimento de tal atividade. Já para a atividade proteolítica, em apenas um ponto de colheita houve diferença quanto ao período, chuva e seca, sendo melhor na seca, e em outros três pontos houve diferença quanto ao tipo de ordenha, sendo em duas a manual e em uma a mecânica com melhor condição para não desenvolvimento da atividade enzimática. Mesmo não havendo diferenças significativas de presença de atividade enzimática em alguns pontos de colheita, os números, em porcentagem de presença de atividade, apresentados são consideravelmente altos $(37 \pm 11 \%$ lipolítica e $29 \pm 14 \%$ proteolítica - média e desvio padrão da presença de atividade enzimática do total de amostras), pois a atividade enzimática pode comprometer a qualidade do produto final. Desta forma, os resultados sugerem que os procedimentos relacionados à higienização de todo sistema de obtenção de leite nas ordenhas, em associação com o tempo e a temperatura de estocagem do leite no local, não foram suficientes para reduzir a contaminação e a multiplicação dos micro-organismos deteriorantes.
\end{abstract}

PALAVRAS-CHAVE: LEITE. PROTEOLÍTICA. LIPOLÍTICA.

ÁREA TEMÁTICA: Higiene e Inspeção de Produtos de Origem Animal.

\footnotetext{
${ }^{1}$ Graduanda em Engenharia de Alimentos, Universidade de São Paulo, Faculdade de Zootecnia e Engenharia de Alimentos, Pirassununga. fernanda.bortoletto@usp.br

${ }^{2}$ Profa. Doutora, Departamento de Medicina Veterinária, Universidade de São Paulo, Faculdade de Zootecnia e Engenharia de Alimentos, Pirassununga. anavidal@usp.br

${ }^{3}$ Prof. Associado, Departamento de Zootecnia, Universidade de São Paulo, Faculdade de Zootecnia e Engenharia de Alimentos, Pirassununga. saranetto@usp.br

${ }^{4}$ Biologa, Assistente técnica do Departamento de Medicina Veterinária, Universidade de São Paulo, Faculdade de Zootecnia e Engenharia de Alimentos, Pirassununga. ancvaz@usp.br

${ }^{5}$ Pós-Graduando (nível mestrado) em Qualidade e Produtividade Animal, Departamento de Zootecnia, Universidade de São Paulo, Faculdade de Zootecnia e Engenharia de Alimentos, Pirassununga. ecapo@ usp.br. Endereço de trabalho: Avenida Duque de Caxias Norte, 225, Campus da USP, ZMV, Cep 13635-900, Pirassununga, São Paulo, Brasil.

${ }^{6}$ Unesp - Universidade Estadual Paulista "Julio de Mesquita Filho" (UNESP), Faculdade de Ciência Agrárias e Veterinárias (FCAV), Via de acesso Paulo Castellane, s/n, Jaboticabal, São Paulo, Brasil, CEP 14884-900,

Correio eletrônico do autor responsável: anavidal@usp.br
} 